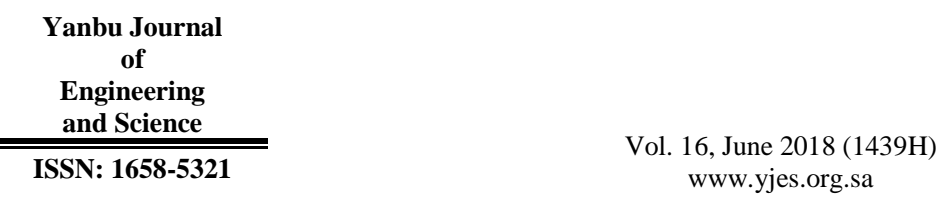

\title{
NUMERICAL SOLUTION OF CONVECTION-DIFFUSION EQUATION BY CHEBYSHEV SPECTRAL METHOD VIA LIE GROUP METHOD
}

\author{
M. A. Assabaai \\ Department of Mathematics, Faculty of Science, Hadhramout University, Yemen. \\ E-mail: asaba973@gmail.com
}

\begin{abstract}
The numerical solution of convection-diffusion equation is presented by using Chebyshev spectral method based on El-Gendi method via Lie group analysis. Firstly, we apply Lie symmetry group analysis for the convection-diffusion equation. This method yields convection-diffusion equation to a system of ordinary differential equations (ODEs). Secondly, this system is solved numerically by using Chebyshev spectral method. The numerical results obtained by this way are compared with the exact solution.
\end{abstract}

Keywords: Chebyshev spectral method, Convection-diffusion equation, Lie group analysis.

\section{INTRODUCTION}

In this paper, we will consider the convection-diffusion equation;

$$
\frac{\partial u}{\partial t}+\alpha \frac{\partial u}{\partial x}-\varepsilon \frac{\partial^{2} u}{\partial x^{2}}=0,0 \leq x \leq 1,0 \leq t \leq T
$$

with initial condition

$$
u(x, 0)=f(x), \quad 0 \leq x \leq 1
$$

and perturbed boundary conditions

$$
\begin{aligned}
& u(0, t)=g_{0}(t)+\varepsilon_{0}, \quad 0 \leq t \leq T, \\
& u(1, t)=g_{1}(t)+\varepsilon_{1}, \quad 0 \leq t \leq T
\end{aligned}
$$

Where $f, g_{0}$ and $g_{1}$ are known functions, the parameters $\alpha$ and $\varepsilon$ are convection and diffusion coefficients respectively and both are assumed to be positive, and $\varepsilon_{0}, \varepsilon_{1}$ are small positive numbers. A large number of problems in physics, chemistry, financial mathematics and many other fields can be modelled by convection-diffusion equation, (for example see $[1,11]$ ), for more applications see [13, 25, 29, 33 and 34].
There are many researchers who used various numerical methods to solve convection-diffusion equation. Appadu [2] solved the convection-diffusion equation using standard and nonstandard finite difference schemes. Chawla et al. [9] presented extended one-step timeintegration schemes for convectiondiffusion equations. El-Hawary and AbdelRahman [16] studied numerical solution of convection-diffusion equation (linear Burger's) by a spectral/spline method. ELWakil and Elhanbaly [17] solved the convection-diffusion equation using Adomian decomposition method. Feng [19], explicit finite difference method was used to solve convection-diffusion equation. Olayiwola [27] presented Variational iteration method to solve convection-diffusion equation. In Porshokouhi et al. [30], Homotopy perturbed method was used to solve convection-diffusion equation. Temsah [31] presented steady-state solution for 
convection-diffusion equation using ElGendi method. For more applications to obtain the numerical solutions of convection-diffusion equation see $[4,8,10$, 12, 14, 18, 20, 26 and 32]. In this work, we use the spectral method based on expanding the unknown functions in terms of Chebyshev polynomials of order $\mathrm{N}$. The purpose of this paper is to find a numerical solution of convection-diffusion equation by using Chebyshev expansion method, which is a continuations of our previous work [3], in which we found the exact solution of the given equation by using lie group method. For the applications of lie group method refer to $[6,7,21,22,23,24$ and 28].

\section{PROBLEM DEFINITION}

\subsection{METHOD OF CHEBYSHEV EXPANSION}

The Chebyshev polynomial of the first kind of degree $n, \mathrm{~T}_{n}(x)$, has $(n+1)$ extreme located in the interval $[-1,1]$ at the points

$$
x_{i}=\cos (i \pi / N), i=0,1, \cdots, N
$$

Assume that the function $f(x)$ is defined and well-behaved in the interval $(-1,1)$. We have the approximation

$$
f(x)=\sum_{r=0}^{N} a_{r} \mathrm{~T}_{r}(x)
$$

where, the Chebyshev coefficients $a_{r}$ are defined as

$$
a_{r}=\frac{2}{N} \sum_{i=0}^{N} \prime \prime f\left(x_{i}\right) \mathrm{T}_{r}\left(x_{i}\right), \quad r=0,1, \cdots, N
$$

Here $\mathrm{T}_{r}(x)$ is the $r^{\text {th }}$ Chebyshev polynomial. A double prime indicates that both the first and last terms of the sum are taken with factor $\frac{1}{2}$. The approximate formula (5) is exact at $x=x_{i}$ given by (4).
If we use (6) in (5), then after certain manipulations we have the following approximation

$$
\int_{-1}^{x} f(x) d x=B[f]
$$

where $B$ is an $(N+1) \times(N+1)$ matrix (see [15]), the elements of the column $[f]$ are given by $f\left(x_{i}\right)$ where $x_{i}$ are the Chebyshev points

$$
x_{i}=-\cos (i \pi / N), i=0,1, \cdots, N
$$

The right-hand side of equation (7) gives approximations of the integral at the points (4). In other words, we evaluate the integral at the points (8) rather than evaluate the Chebyshev coefficients of the integral. The two approximations are equivalent in the sense that if we know the integral at the points (8), its Chebyshev coefficients can be directly evaluated from (6). We note that the computation of the elements of $B$ does not depend on the integral. Note: For the interval $(0,1)$ the elements of matrix $B$ are to be halved, and the Chebyshev points will be $x_{i}=\frac{1}{2}(1-\cos (i \pi / N)), i=0,1, \cdots, N$

such that the Chebyshev polynomials are given by

$$
\mathrm{T}^{*}{ }_{r}(x)=\mathrm{T}_{r}(2 x-1), \quad 0 \leq x \leq 1
$$

where, all the properties of $\mathrm{T}^{*}{ }_{r}(x)$ can be deduced from those of $\mathrm{T}_{r}(2 x-1)$.

\section{LIE GROUP TRANSFORMATION METHOD}

The aim of this method is used to transform the original partial differential equation (PDE) to ordinary differential equation (ODE). [3] presented the method of Lie group of transformations, which makes convection-diffusion equation invariant. The one-parameter $\varepsilon$-Lie group point of transformations are given by 
$\bar{x}=\bar{x}(x, t, u ; \varepsilon)=x+\varepsilon \xi_{1}(x, t, u)+\mathrm{O}\left(\varepsilon^{2}\right)$,

$\bar{t}=\bar{t}(x, t, u ; \varepsilon)=t+\varepsilon \xi_{2}(x, t, u)+O\left(\varepsilon^{2}\right)$,

$\bar{u}=\bar{u}(x, t, u ; \varepsilon)=u+\varepsilon \varphi(x, t, u)+O\left(\varepsilon^{2}\right)$

The infinitesimal generator for (10) is given by

$\mathrm{X}=\xi_{1} \frac{\partial}{\partial x}+\xi_{2} \frac{\partial}{\partial t}+\varphi \frac{\partial}{\partial u}$

and the second prolongation of the infinitesimal generator (11) is given by

$\operatorname{Pr}^{(2)} X=X+\varphi^{x} \frac{\partial}{\partial u_{x}}+\varphi^{t} \frac{\partial}{\partial u_{t}}+\varphi^{t \prime} \frac{\partial}{\partial u_{t t}}+\varphi^{x} \frac{\partial}{\partial u_{x t}}+\varphi^{t} \frac{\partial}{\partial u_{t t}}$

The components $\varphi^{x}, \varphi^{t}, \varphi^{x t}, \varphi^{x x}$ and $\varphi^{t t}$ can be determined from the following expressions:

$$
\begin{aligned}
\varphi^{\alpha} & =D_{\alpha} \varphi-u_{x} D_{\alpha} \xi_{1}-u_{t} D_{\alpha} \xi_{2}, \\
\varphi^{J \alpha} & =D_{\alpha} \varphi^{J}-u_{J x} D_{\alpha} \xi_{1}-u_{J t} D_{\alpha} \xi_{2}, \\
D_{\alpha} & \equiv \frac{\partial}{\partial \alpha}+u_{\alpha} \frac{\partial}{\partial u}+u_{\alpha \alpha} \frac{\partial}{\partial u_{\alpha}}+u_{\alpha \beta} \frac{\partial}{\partial u_{\beta}}+\ldots
\end{aligned}
$$

where $\alpha, J$ and $\beta$ stand for independent variables ${ }^{x, t}$, considered

$$
\Delta=\frac{\partial u}{\partial t}+\alpha \frac{\partial u}{\partial x}-\varepsilon \frac{\partial^{2} u}{\partial x^{2}}
$$

To calculate the infinitesimals $\xi_{1}, \xi_{2}$ and $\varphi$, applying (12) to (14), i. e.,

$\operatorname{Pr}^{(2)} \mathrm{X}(\Delta)=0$, when $\Delta=0$

Equation (15) is called invariance condition, and leads to

$\varphi^{t}+\alpha \varphi^{x}-\varepsilon \varphi^{x x}=0$,

Where $\varphi^{t}, \varphi^{x}$ and $\varphi^{x x}$ are obtained from (13). The infinitesimals solutions $\xi_{1}, \xi_{2}$ and $\varphi$ are given by

$$
\begin{aligned}
\xi(x, t, u)= & \frac{1}{2} x t c_{1}+\frac{1}{2}(\alpha t+x) c_{2}-2 \varepsilon t c_{5}+c_{8}, \\
\xi_{2}(x, t, u)= & -t^{2} c_{1}+t c_{2}+c_{3}, \\
\varphi(x, t, u)= & c_{6} \exp \left(t+\frac{1}{2 \varepsilon}\left(\alpha-\sqrt{\alpha^{2}+4 \varepsilon}\right) x\right)+c_{7} \exp \left(a t+\frac{1}{2 \varepsilon}\left(\alpha+\sqrt{\alpha^{2}+4 \varepsilon}\right) x\right) \\
& +u c_{4}-(\alpha t-x) u c_{5}-\frac{1}{4}\left(t-\frac{1}{2 \varepsilon}(\alpha t-x)^{2}\right) u c_{1}
\end{aligned}
$$

where $c_{i}, i=1,2, \ldots, 8$ are arbitrary constants. From equation (17), the convectiondiffusion equation has the following generators

$$
\begin{aligned}
& \mathrm{X}_{1}=\frac{1}{2} x t \partial_{x}+\frac{1}{2} t^{2} \partial_{t}-\frac{1}{4}\left(t-\frac{1}{2 \varepsilon}(\alpha t-x)^{2}\right) u \partial_{u}, \\
& \mathrm{X}_{2}=\frac{1}{2}(\alpha t+x) \partial_{x}+t \partial_{t}, \\
& \mathrm{X}_{3}=\partial_{t}, \mathrm{X}_{4}=u \partial_{u}, \mathrm{X}_{5}=-2 \varepsilon t \partial_{x}-(\alpha t-x) u \partial_{u}, \\
& \mathrm{X}_{6}=\exp \left(t+\frac{1}{2 \varepsilon}\left(\alpha-\sqrt{\alpha^{2}+4 \varepsilon}\right) x\right) \partial_{u}, \\
& \mathrm{X}_{7}=\exp \left(t+\frac{1}{2 \varepsilon}\left(\alpha+\sqrt{\alpha^{2}+4 \varepsilon}\right) x\right) \partial_{u}, \mathrm{X}_{8}=\partial_{x}
\end{aligned}
$$

As in [3], we use the linear combination of generators $\mathrm{X}_{3}$ and $\mathrm{X}_{8}$ as follows

$$
\mathrm{X}_{3,8}=\partial_{t}+c \partial_{x},
$$

and getting of the following similarity reductions:

$$
\begin{aligned}
& \eta=x-c t, \\
& f(\eta)=u(x, t)
\end{aligned}
$$

Equation (20) reduce the original equation (1) to the ordinary differential equation:

$$
\varepsilon f^{\prime \prime}(\eta)+(c-\alpha) f^{\prime}(\eta)=0, \quad \eta \in[-c t, 1-c t]
$$

In this work, without loss of the generality, we can choose $c=1$, and equation (21) becomes

$$
\varepsilon f^{\prime \prime}(\eta)+(1-\alpha) f^{\prime}(\eta)=0, \quad \eta \in[-t, 1-t]
$$

with the moving boundary conditions

$$
f=g_{0}(t)+\varepsilon_{0} a t \eta \rightarrow-t, \quad f=g_{1}(t)+\varepsilon_{1} a t \eta \rightarrow 1-t
$$

Using the algebraic mapping, $y=\eta+t$,

the boundary region is mapped into the finite domain $(0,1)$, and the problem expressed by equations (22) with (23) is transformed to

$$
\begin{aligned}
& \varepsilon f^{\prime \prime}+(1-\alpha) f^{\prime}=0 \\
& f(0)=g_{0}(t)+\varepsilon_{0}, \\
& f(1)=g_{1}(t)+\varepsilon_{1}
\end{aligned}
$$


where the prime denotes differentiation with respect to $y$.Our technique is accomplished by starting with a Chebyshev approximation for the higher order derivatives $f^{\prime \prime}$ and generating approximations to the lower derivatives $f^{\prime}$ and $f$ as follows. Setting $\psi(y)=f^{\prime \prime}(y)$, then by integration we obtain

$$
\begin{aligned}
& f^{\prime}(y)=\int_{0}^{y} \psi(y) d y+c_{1}, \\
& f(y)=\int_{00}^{y y} \psi(y) d y d y+y c_{1}+c_{2}
\end{aligned}
$$

From equation (27), we get

$c_{1}=g_{1}(t)+\varepsilon_{1}-\left(g_{0}(t)+\varepsilon_{0}\right)-\int_{00}^{1} \int_{0}^{y} \psi(y) d y d y$,

$c_{2}=g_{0}(t)+\varepsilon_{0}$

we give Chebyshev approximations for equations (26) and (27) as follows:

$$
\begin{aligned}
& f^{\prime}\left(y_{i}\right)=\sum_{j=0}^{N} \ell_{i j}^{(1)} \psi\left(y_{j}\right)+\stackrel{(1)}{d}_{i}, \\
& f\left(y_{i}\right)=\sum_{j=0}^{N} \ell_{i j} \psi\left(y_{j}\right)+d_{i}
\end{aligned}
$$

for $\quad$ all $\quad i, j=0,1, \ldots, N \quad$ and $y_{i}=1 / 2(1-\cos (i \pi / N)) \quad$ where

$\ell_{i j}=\stackrel{(2)}{b}_{i j}-y_{i}{\stackrel{(2)}{b_{N}}}, \stackrel{(1)}{\ell}_{i j}=b_{i j}-{\stackrel{(2)}{b_{N j}},}$

$d_{i}=\left(g_{1}(t)+\varepsilon_{1}-\left(g_{0}(t)+\varepsilon_{0}\right)\right) y_{i}+g_{0}(t)+\varepsilon_{0}$,

$\stackrel{(1)}{d}_{i}=g_{1}(t)+\varepsilon_{1}-\left(g_{0}(t)+\varepsilon_{0}\right)$

and $\stackrel{(2)}{b_{i j}}=\left(y_{i}-y_{j}\right) b_{i j}, \quad i, j=0,1, \ldots, N$.

where, $b_{i j}$ are the elements of the matrix $\frac{1}{2} \mathrm{~B}$ as defined by El-Gendi [15] by using equations (28) and (29). Equation (24) is transformed to the following nonlinear system:

$$
\psi_{i}+\frac{1-\alpha}{\varepsilon}\left(\sum_{j=0}^{N} \stackrel{(1)}{\ell}_{i j} \psi\left(y_{j}\right)+\stackrel{(1)}{d}\right)=0
$$

This system is solved by using Gauss elimination method to obtain the approximate solution of the original system.

\section{RESULTS AND DISCUSSION}

\subsection{NUMERICAL EXAMPLES}

In this section, we present some examples of convection-diffusion equation and we compare our numerical results with the exact solutions, see Tables 1 to 3 . Computations of the examples have been carried out using the FORTRAN. In Table 1 , we present absolute errors for various values of $x, t$ with $\varepsilon_{0}=0, \varepsilon_{1}=0$ ( without perturbations) and $\mathrm{N}=18$ for example 1.The maximum absolute error for various values of $t$ with $\varepsilon=1, \varepsilon_{0}=0, \varepsilon_{1}=0$ and $\mathrm{N}=12$ are shown in Table 2 for example 2. Table 3 shows absolute errors in the results obtained by the proposed method for various values of $x, t$ with $\varepsilon_{0}=10 E-15$, $\varepsilon_{1}=10 E-13$ and $\mathrm{N}=18$ for example 3 . Example 1, consider the convectiondiffusion equation in (2)

$$
\frac{\partial u}{\partial t}+\frac{\partial u}{\partial x}-0.01 \frac{\partial^{2} u}{\partial x^{2}}=0
$$

where, $f(x)=\exp \left(-\frac{(x+0.5)^{2}}{0.00125}\right)$

$$
\begin{aligned}
& g_{0}(t)=\frac{0.025}{\sqrt{0.00025+0.02 t}} \exp \left(-\frac{(0.5-t)^{2}}{(0.00125+0.04 t)}\right) \\
& g_{1}(t)=\frac{0.025}{\sqrt{0.00025+0.02 t}} \exp \left(-\frac{(1.5-t)^{2}}{(0.00125+0.04 t)}\right)
\end{aligned}
$$

The exact solution is

$$
u(x, t)=\frac{0.025}{\sqrt{0.00025+0.02 t}} \exp \left(-\frac{(x+0.5-t)^{2}}{(0.00125+0.04 t)}\right)
$$

Example 2, consider the convectiondiffusion equation in (5) 
$\frac{\partial u}{\partial t}+\frac{\partial u}{\partial x}-\varepsilon \frac{\partial^{2} u}{\partial x^{2}}=0$, and the exact solution

is

$u(x, t)=\frac{1}{\sqrt{s}} \exp \left(-50 \frac{(x-t)^{2}}{s}\right), \quad s=1+200 \varepsilon t$

where, the initial condition $u(x, 0)$ and boundary conditions $u(x, t)$ at $x=0,1$ without perturbations are obtained from the exact solution. Example 3, consider the convection-diffusion equation in (30)

$$
\frac{\partial u}{\partial t}+0.22 \frac{\partial u}{\partial x}-0.5 \frac{\partial^{2} u}{\partial x^{2}}=0
$$

where, $f(x)=\sin (\pi x) e^{0.22 x}$

$$
g_{0}(t)=0=g_{1}(t)
$$

The exact solution is

$$
u(x, t)=\sin (\pi x) e^{0.22 x-\left(0.0242+0.5 \pi^{2}\right) t} .
$$

TABLE 1: THE ABSOLUTE ERRORS BETWEEN EXACT AND NUMERICAL SOLUTION FOR EXAMPLE 1.

\begin{tabular}{|c|c|c|c|c|}
\hline \multirow{2}{*}{$x$} & \multicolumn{4}{|c|}{$t$} \\
\cline { 2 - 5 } & 0.01 & 0.05 & 0.08 & 0.1 \\
\hline 0.25 & $0.000000 \mathrm{E}+00$ & $4.052167 \mathrm{E}-28$ & $2.419396 \mathrm{E}-18$ & $2.127136 \mathrm{E}-14$ \\
\hline 0.5 & $0.000000 \mathrm{E}+00$ & $2.701445 \mathrm{E}-28$ & $1.612931 \mathrm{E}-18$ & $1.418091 \mathrm{E}-14$ \\
\hline 0.75 & $0.000000 \mathrm{E}+00$ & $1.350722 \mathrm{E}-28$ & $8.064654 \mathrm{E}-19$ & $7.090453 \mathrm{E}-15$ \\
\hline
\end{tabular}

TABLE 2: THE MAXIMUM ABSOLUTE ERRORS FOR EXAMPLE 2

\begin{tabular}{|c|c|c|c|c|c|}
\hline$t$ & 1.0 & 1.5 & 2.0 & 2.5 & 3.0 \\
\hline & $3.514044 \mathrm{E}-03$ & $1.338173 \mathrm{E}-03$ & $5.243644 \mathrm{E}-04$ & $1.703687 \mathrm{E}-04$ & $3.712066 \mathrm{E}-05$ \\
\hline
\end{tabular}

TABLE 3: THE ABSOLUTE ERRORS BETWEEN EXACT AND NUMERICAL SOLUTION FOR EXAMPLE 3

\begin{tabular}{|c|c|c|c|c|c|c|c|}
\hline \multirow{2}{*}{$x$} & \multicolumn{7}{|c|}{$t$} \\
\cline { 2 - 8 } & 1 & 2 & 3 & 4 & 5 & 6 & 7 \\
\hline 0.25 & $5.24450 \mathrm{E}-03$ & $3.68160 \mathrm{E}-05$ & $2.58445 \mathrm{E}-07$ & $1.81426 \mathrm{E}-09$ & $1.27318 \mathrm{E}-11$ & $8.51697 \mathrm{E}-14$ & $3.60866 \mathrm{E}-15$ \\
\hline 0.5 & $7.83619 \mathrm{E}-03$ & $5.50095 \mathrm{E}-05$ & $3.86163 \mathrm{E}-07$ & $2.71083 \mathrm{E}-09$ & $1.90225 \mathrm{E}-11$ & $1.26259 \mathrm{E}-13$ & $6.39146 \mathrm{E}-15$ \\
\hline 0.75 & $5.85432 \mathrm{E}-03$ & $4.10969 \mathrm{E}-05$ & $2.88497 \mathrm{E}-07$ & $2.02522 \mathrm{E}-09$ & $1.42077 \mathrm{E}-11$ & $9.05707 \mathrm{E}-14$ & $8.53068 \mathrm{E}-15$ \\
\hline
\end{tabular}

\section{CONCLUSION}

The numerical results obtained by Chebyshev approximation method based on El-Gendi method via Lie group method for convection- diffusion equation show reasonable agreement with the exact solution as shown in Tables 1-3.
Consequently, the presented method performs well.

\section{REFERENCES}

[1] Anderson, D. A. Tannehill, J. C. and Pletcher, R. H., Computational Fluid Mechanics and Heat Transfer, Series in Computational 
Methods in Mechanics and Thermal Sciences, Mc Graw-Hill,1997.

[2] Appadu, A. R., Numerical Solution of the 1D Advection-Diffusion Equation Using Standard and Nonstandard Finite Difference Schemes, Journal of Applied Mathematics Volume 2013, Article ID 734374, 14 pages, 2013.

[3] Assabaai, M. A. and Bamsaoud, S. F., Exact solutions of the one-dimensional convectiondiffusion equation using Lie group method, Assiut Univ. J. of Mathematics and Computer Science, 46 (1), p 1-12, 2017.

[4] Baza'n, F. S. V., Chebyshev pseudospectral method for computing numerical solution of convection-diffusion equation, Appl. Math. and compt. 200, p537-546, 2008.

[5] Berzins, M., Capon, P.J. and Jimack, P. K., On spatial adaptivity and interpolation when using the method of lines, Appl. Numer. Math., 26, p117-133, 1998.

[6] Bluman, G. and Kumei, S., Symmetries and Differential Equations, Springer-Verlag, 1989.

[7] Bluman, G. W. and Anco, S. C., Symmetry and Integration Methods for Differential Equations, Springer, New York, 2002.

[8] Boztosun, I. and Charafi, A. , An analysis of the linear advection-diffusion equation using mesh-free and mesh-dependent methods, Engineering Analysis with Boundary Elements 26, 889-895, 2002.

[9] Chawla, M. M., AL-Zanaidi, M. A. and ALAslab, M. G., Extended one-step timeintegration schemes for convection-diffusion equations, Comput. Math. Appl., 39, p71-84, 2000.

[10] Chawla, M. M., Karaballi, A. A. and ALSahhar, M. S., Extended double-stride Lstable methods for the numerical solution of ODEs, Comput. Math. Appl., 31 (2), p1-6, 1996.

[11] Chernesky, M. P., On preconditioned Krylov subspace methods for discrete convectiondiffusion problems, Numer. Methods Partial Differential Equations, 13 (4), p321-330, 1997.

[12] Crank, J. and Nicolson, P., A practical method for numerical evaluation of solutions of partial differential equations of the heat-conduction type, Proc. Camb. Philos. Soc., 43, p50-67, 1947.

[13] Daga, A. and Pradhan, V. H., Analytical Solution of Advection Diffusion Equation in Homogeneous Medium, PRATIBHA: International Journal of Science, Spirituality, Business and Technology (IJSSBT), 2 (1), p2277-7261, 2013.
[14] Dehghan, M., On the numerical solution of the one-dimensional convection-diffusion equation, Mathematical Problems in Engineering, 1, p61-74, 2005.

[15] EL-Gendi, S. E., Chebyshev solution of differential integral and integro differential equations, Comput. J., 12, p282-287, 1969.

[16] El-Hawary, H. M. and Abdel-Rahman, E. O., Numerical solution of the generalizedBurger's equation via spectral/spline methods, Applied Mathematics and Computation, 170, p267279, 2005.

[17] EL-Wakil, S. A. and Elhanbaly, A., Adomian decomposition method for solving the diffusion-diffusion reaction equations, Appl. Math. and compt., 177, p729-736, 2006.

[18] Fallahzadeh, A. and Shakibi, K., A method to solve Convection Diffusion equation based on Homotopy analysis method. Journal of Interpolation and Approximate in Scientific Computing, p1-8, 2015.

[19] Feng, Q., Explicit Finite Difference Method for Convection-Diffusion Equation, Proceedings of the World Congress on Engineering, Vol II, London, U. K, 2009.

[20] Ghasemi, M. and Kajani, M. T., Application of He's homotopy perturbation method to solve a diffusion-convection problem, Mathematical Sciences, 4, p171-186, 2010.

[21] Hamad, M. A. A., Hassanien, I. A. and ElNahary, E. Kh. H., Discrete Symmetries Analysis of Burgers Equation with Time Dependent Flux at the Origin, World Applied Sciences Journal, 12, p2291-2300, 2011.

[22] Hizel, E. and Turgay, N. C., Symmetry group analysis and similarity solutions for nonlinear Reaction-Diffusion system of Gray-Scott type, Int. Math. Forum., 2, p2847-2858, 2007.

[23] Hydon, P. E., symmetry methods for differential equations. A beginners' guide, Cambridge Texts in Applied Mathematics, 2000.

[24] Ibragimov, N. H. (Ed.), CRC Handbook of Lie groups analysis of Differential Equations, II. Application in engineering and physical sciences, CRC Press, Boca Raton, 1994.

[25] Kumer, N., Unsteady flow against dispersion in finite porous media, J. Hydrol., 63 (3-4), p345-358, 1983.

[26] Mohammadi, A., Manteghian, M. and Mohammadi, A., Numerical solution of the one- dimensional advection-diffusion equation using simultaneously temporal and spatial weighted parameters, Australian 
Journal of Basic and Applied Sciences, 5(6), p1536-1543, 2011.

[27] Olayiwola, O., Application of Variational Iteration Method to the Solution of Convection-Diffusion Equation, J. Appl. Computat. Math., 5: 299. doi:10.4172/21689679.1000299, 2016.

[28] Olver, P. J., Applications of Lie Groups to Differential Equations, Grad. Texts in Math., vol. 107, Springer-Verlag, New York, 1993.

[29] Parlange, J. Y., Water transport in soils, Annu. Rev. Fluid Mech., 12, p77-102, 1980.

[30] Porshokouhi, M. G., Ghanbari, B., Gholami, M. and Rashidi, M., Approximate solution of Convectional-Diffusion equation by the Homotopy perturbation method, Gen. Math. Notes, 1(2), p108-114, 2010.

[31] Temsah, R. S., Numerical solutions for convection-diffusion equation using El-Gendi method, Communications in Nonlinear Science and Numerical Simulation, 14, 760769, 2009.

[32] Usmani, R. A. and Agarwal, R. P., An astable extended trapezoidal rule for the numerical integration of ordinary differential equations, Comput. Math. Appl., 11 (12), p1183-1191, 1985.

[33] Veling, E. D., Analytical solution and numerical evaluation of the radial symmetric convection-diffusion equation with arbitrary initial and boundary data, Impact of Human Activityon Groundwater Dynamics (Proceedings of a symposium held during the Sixth IAHS Scientific Assembly at Maastricht, The Netherlands, IAHS Publ. no. 269, 2001.

[34] Zlatev, Z. Berkowicz, R. and Prahm, L. P., Implementation of a variable step size variable formula method in the timeintegration part of a code for treatment of long-range transport of air pollutants, J. Comput. Phys., 55 (2), p278-301, 1984. 
Yanbu Journal of Engineering and Science Vol. 16 (2018)

- 64 - 
